\title{
Modélisation et Cartographie des Risques d'érosion Hydrique du Sol par l'application des SIG, Télédétection et Directives PAP/CAR. Cas du Bassin Versant de Beht, Maroc
}

\author{
Dr. Ait Yacine E., \\ Pr. Oudija F., \\ Pr. Nassiri L.,
}

Université Moulay Ismail, Faculté des Sciences, Département de Biologie, Laboratoire de Microbiologie du Sol et de l'Environnement, Zitoune, Meknès, Maroc

Pr. Essahlaoui A.,

Université Moulay Ismail, Faculté des Sciences, Département de Géologie, équipe d'hydrogéologie et ingénierie de l'environnement,

Zitoune, Meknès, Maroc

Doi: 10.19044/esj.2019.v15n12p259 URL:http://dx.doi.org/10.19044/esj.2019.v15n12p259

\section{Résumé}

La modélisation et la cartographie des potentiels risques de l'érosion hydrique des sols est d'une grande importance dans la gestion et l'aménagement des bassins versants. Ce travail a pour objectif d'élaborer la carte de vulnérabilité des sols à l'érosion dans le bassin versant de Beht en amont du barrage de Ouljet Soltane, en se basant sur les directives du Programmes d'Actions Prioritaires/ Centre d'Activité Régionale (PAP /CAR).Cette méthode consiste à définir les principaux facteurs causaux (pente, lithologie, érodibilité, occupation du sol, densité du couvert végétal, formes et ampleur d'érosion existant sur terrain) et fait appel à un ensemble de données ( MNT, Géologie, images Landsat 8,et autres..) en utilisant SIG et télédétection. Le bassin versant de Beht, qui est un affluent principal de l'Oued Sebou, couvre deux unités géomorphologiques dont le moyen Atlas et le plateau central. L'application de la méthode PAP/CAR, qui comporte trois phases, a donné les résultats suivants: dans la $1{ }^{\text {ère }}$ phase (prédictive) la carte des états érosifs, montre que 59\% des terrains du bassin d'étude sont exposé à des fortes à très fortes érosions. Les résultats obtenus pour la $2^{\text {ème }}$ phase (descriptive), montrent que ce bassin versant est réellement touché par l'érosion à différentes formes et que seulement $21 \%$ de la surface peut être considérée comme stable ou stabilisée mais avec un risque d'instabilité 
non nul. Pour la $3^{\text {ième }}$ phase, qui est celle d'intégration, elle a permis d'obtenir la carte consolidée de l'érosion qui montre que le degré d'intensité de l'érosion est proportionnel à celle des facteurs causaux et surtout dans les zone de combinaison des facteurs (pente et friabilité du sol à leurs haut niveau et le couvert végétal et sa densité à leurs bas niveaux).

Mots-clés : Érosion, PAP/CAR, bassin versant de Beht, SIG

\title{
Modeling and Mapping Water Erosion Risk by Application of GIS, Remote Sensing and PAP/RAC Guidelines. Case of the Beht Watershed, Morocco
}

\author{
Dr. Ait Yacine E., \\ Pr. Oudija F., \\ Pr. Nassiri L.,
}

Université Moulay Ismail, Faculté des Sciences, Département de Biologie, Laboratoire de Microbiologie du Sol et de l'Environnement,

Zitoune, Meknès, Maroc

Pr. Essahlaoui A.,

Université Moulay Ismail, Faculté des Sciences, Département de Géologie, équipe d'hydrogéologie et ingénierie de l'environnement,

Zitoune, Meknès, Maroc

\begin{abstract}
Modeling and mapping potential risk of soil erosion is extremely important to watershed management. The advanced technology of geomatics as Geographic Information System (GIS) and Remote sensing (RS) become a valuable source of assistance to estimate soil loss at the large area, in faster manner, and with a consistent level of reliability.The main objective of this work is to elaborate the map of vulnerable area to the hydrical erosion based on the The Priority Actions Programme / Regional Activity Centre (PAP/RAC) method using (GIS) and (RS) in the Beht watershed in upstream of Ouljat Sultan dams. This watershed occupies the upstream part of El Kansera basin and it is one of sub-watersheds of the Sebou River. It extends an area of approximately $2472 \mathrm{~km}^{2}$ and overlaps on two geomorphologicals units: the Central Meseta and the Tabular Middle Atlas. En deed, the PAP/RAC method is based on three approaches: the first
\end{abstract}


is the predictive approach that provides a synthetic map of erosive states, the result of this step shows that $59 \%$ of study basin is subjected to a high and very high degree of erosion. The second is the descriptive approach that allows to maps the various erosion forms. Result of this approach hows that only $21 \%$ of study watershed is stable or stabilized by vegetation; the rest is infected by all erosion forms with different degree. The third approach provides a consolidated and integrated map of erosion, which shows that the degree of erosion intensity is proportional to that of the causal factors and especially in the area who combine the causal factors, (slope and friability of soil at their high levels, and the vegetation cover and its density at their low levels).

Keywords: Water erosion, PAP/RAC method, Geographic Information System(GIS), Watershed

\section{Introduction}

L'érosion hydrique du sol est un phénomène qui touche les pays méditerranéens, et essentiellement le Maroc. L'Organisation Mondiale de l'Alimentation et l'Agriculture (FAO) en 1990, rapporte que ce phénomène affecte $35 \%$ des terres en Grèce, $40 \%$ au Maroc et $50 \%$ en Turquie (Celik et al, 1996). Au Maroc et selon le Haut-commissariat aux Eaux et Forêt et à la lutte contre la désertification l'érosion hydrique touche 23 Millions ha, et la dégradation spécifique des sols varie de 500 t/km2/an au Moyen Atlas, à plus de 5000 t/km2/an au Rif(HCEFLCD, 1996b) .

Les dégâts causés par ce phénomène sont multiples et nécessitent une veille stratégique : en plus des dommages socio-économiques et des inondations qui menacent les populations et les infrastructures (Roose et al, 2010), ce phénomène réduit la capacité de détention des eaux dans les barrages. Les études menées par le HCEFLCD en collaboration avec la FAO, dans ce sens, ont évalué la réduction de la capacité des barrages par l'érosion hydrique à 75 million $\mathrm{m}^{3} / \mathrm{an}(\mathrm{HCEFLCD}, 1996 \mathrm{~b})$;cela malgré que le pays souffre d'un stress hydrique qui tend à s'aggraver dans les prochaine année. La FAO et l'institut mondial des ressources (WRI) ont classé le Maroc parmi les 19 pays les plus menacés par le stress hydrique à l'horizon 2040 (Haitami, 2017). Selon ces organisations, le Maroc prône une raréfaction extrême en eau, avec une disparition de $80 \%$ des sources en eau actuelles vu les changements climatiques que connaît la Région, et vu les tendances futures du mode de gestion actuel. Le Maroc est appelé donc à entreprendre toutes les mesures pouvant contribuer à la diminution des effets néfastes du stress hydrique. Parmi les solutions envisagées face à ce problème, la construction des barrages est l'une des solutions. Dans ce cadre, il est certes vrai que le Maroc a entrepris depuis les années cinquante une politique de construction 
des barrages, dans l'objectif, à la fois, d'assurer les besoins accrues de la population en eau potable, d'augmenter les superficies irriguées et de diminuer la facture énergétique. Mais la durée de vie de ces édifices couteux et leurs capacités de stockage des eaux se trouvent très limitées par l'envasement dû au phénomène de l'érosion(HCEFLCD, 1996b ; Jaouhari, 2018), d'où la nécessité de lutter contre ce fléau. A ce propos plusieurs modèles et méthodes ont été développés et utilisés dans l'évaluation de l'érosion hydrique du sol (USLE, RUSLE, LEAM, PAP/CAR...) Parmi les méthodes qualitatives les plus utilisées dans le pourtour méditerranéen on trouve la méthode PAP/CAR (PAP/CAR ( PAP/PAM/PNUE), 1998).

Ce modèle qualitatif d'étude de l'érosion hydrique des sols, associé aux systèmes d'information géographique (SIG) et à la télédétection, présente plusieurs avantages en tant qu'outil efficace de prévision de l'érosion spatiale sur de grandes surfaces, de suivi de l'évolution spatio-temporelle et des tendances futures de ce phénomène et une aide à la prise de décision (Tribak et al, 2012).La méthode qualitative PAP/CAR repose sur trois approches (ou phases): la phase prédictive, la phase descriptive et la phase d'intégration.

La zone d'étude qui est le bassin versant de Beht en amont du barrage d'Ouljet Soltane n'est pas choisi d'une façon aléatoire, mais pour des raisons objectives. En effet le bassin versant de Beht en amont du barrage d'el Kansera a été classé par le plan national d'aménagement des bassins versant (PNABV) au huitième ordre de priorité vu l'ampleur de l'érosion enregistré dans ce bassin (HCEFLCD, 1996a).Cela a été approuvé par l'importance des sédiments enregistrés dans les retenues de ce barrage et qui ont diminué sa capacité de stockage, ce qui a poussé le département de l'eau à entreprendre la sur-élevation du barrage d'El Kansera en1969 (SECE. Maroc, 2019b),puis à entamer la construction d'un autre barrage (celui de Ouljet Soltane) en amont de ce dernier (SECE. Maroc, 2019a).Ce travail est d'une grande importance puisqu'il va nous donner une idée sur l'ampleur, les manifestations et les tendances futures de l'érosion dans ce bassin.

\section{Caractéristiques de la Zone d'étude}

Le bassin versant de l'oued Beht en amont du barrage d'Ouljet Soltane constitue la partie amont de l'oued Beht qui est un affluent principal du fleuve de Sebou (Fig.1).Il s'étend sur une superficie d'environ $2472 \mathrm{Km}^{2}$ et se présente en forme allongée selon un axe NO-SE. Le bassin de Beht englobe trois domaines géologiques et structuraux : Le Maroc Central hercynien, le Causse moyen atlasique et le Sillon sud-rifain (Michard, 1976 ; Beaudet, 1969), et il couvre deux unités géomorphologiques, le moyen Atlas tabulaire qui fait la partie Est et Sud-Est et l'amont du bassin d'étude, et la Meseta centrale qui occupe l'ouest et le Nord-Ouest constituant l'aval de ce bassin(Martin, 1982). Du point de vu topographique, le bassin versant de Beht 
est couvert par des terrains plats à très accidentés avec dominance de ces derniers. Les pentes varient de $0 \%$ (sur les terrains plats) à plus de $100 \%$ (sur les falaises comme dans certains endroit sur les rives de l'Oued Beht), et les altitudes entre $299 \mathrm{~m}$ et $2134 \mathrm{~m}$. Dans les parties amont du bassin versant le substratum est à dominance calcaire sous forme d'affleurements de dolomies et de calcaires du Lias inférieur et moyen (Michard, 1976). Les roches basaltiques couvrent également de grandes étendues dans le plateau d'Azrou. Quant au centre et aux parties aval du bassin, le substratum est à base des schistes primaires, associés en plusieurs endroits avec des bancs de quartzites ou de grés de différentes épaisseurs. Les granites, les roches métamorphiques et les calcaires sont peu fréquents.

Le climat régnant dans la zone d'étude est de type méditerranéen avec deux saisons : une saison humide et froide et l'autre sèche et chaude. Il présente un double gradient d'agressivité décroissant du Sud au Nord et de l'Est à l'Ouest avec des précipitations marquées par des irrégularités dans l'espace et dans le temps. Les étages bioclimatiques régnant dans ce bassin varient de l'humide à variante froide, dans le moyen Atlas correspondant à l'amont du bassin, jusqu'à l'aride à variante tempérée dans les vallées de Beht correspondant au plateau centrale constituant l'aval du bassin.

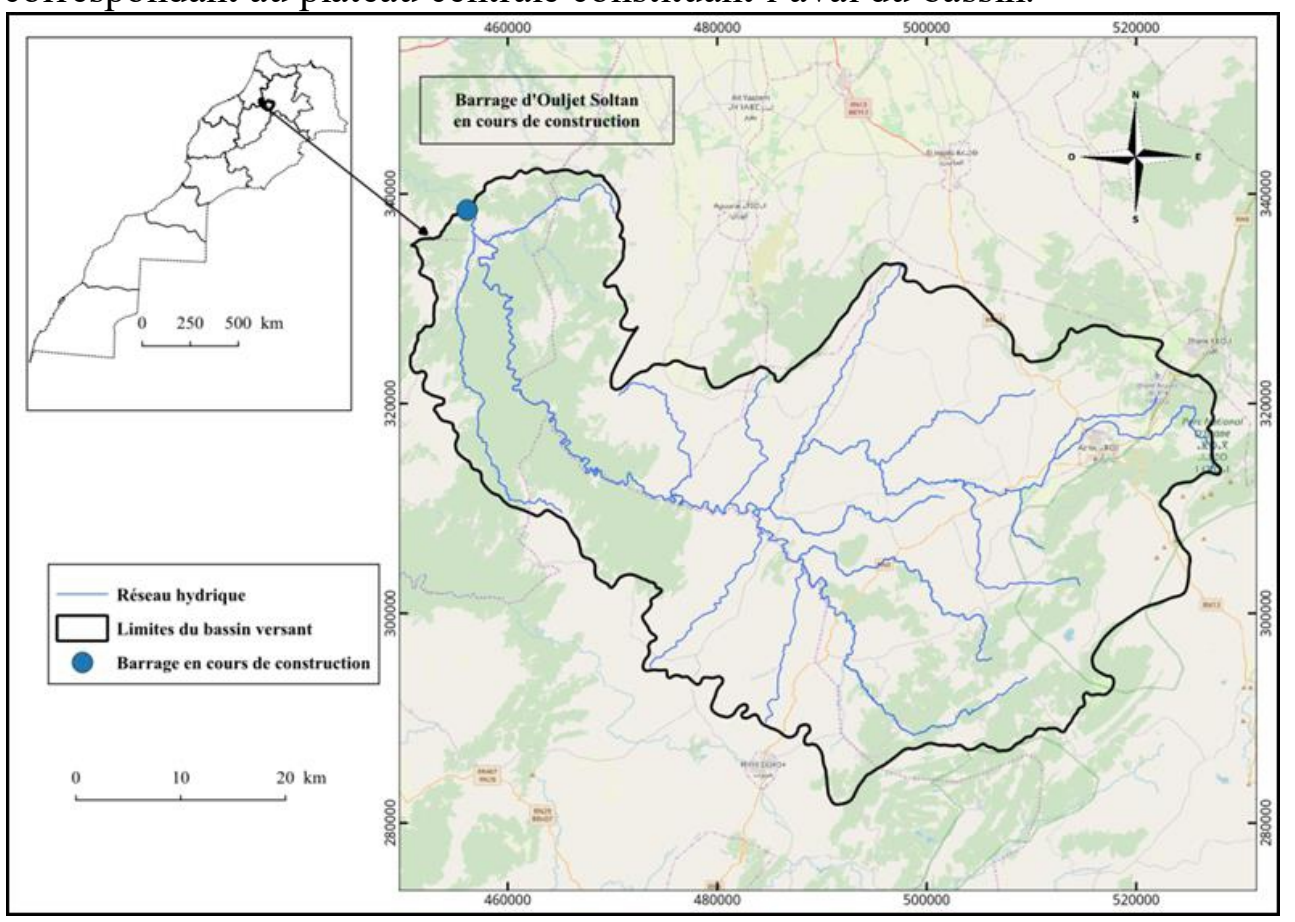

Figure 1: Carte de situation du bassin versant de l'Oued Beht en amont du barrage d'Ouljet Soltane 


\section{Approche Méthodologique et Matériel Utilisé}

La méthode PAP /CAR a été adoptée en 1988 par le Centre d'activités régionales pour le Programme d'actions prioritaires (PAP/CAR) du Plan d'action pour la Méditerranée_PNUE, en collaboration avec la Direction générale de la conservation de la nature (DGCONA) du ministère espagnol de l'Environnement et la Division de la mise en valeur des terres et des eaux (AGL) de l'Organisation des Nations Unies pour l'alimentation et l'agriculture (FAO). Il faut noter aussi que la méthode PAP/CAR a été précédée par une action prioritaire intitulée "Promotion de la protection des sols en tant que composante essentielle de la protection de l'environnement dans les zones côtières méditerranéennes" entrepris par les mêmes coopérants. L'approche méthodologique repose sur 3 phases bien définies répartis en neuf étapes:

- La phase prédictive qui se fait en 7 étapes qui aboutissent à 7 cartes thématiques:

- La carte des pentes:la pente est un facteur principal pour l'évaluation de la vulnérabilité des sols à l'érosion hydrique. La carte des pentes a été synthétisée à partir du modèle numérique du terrain issue d'une image satellitaire ASTER Global Digital Elevation Model (GDEM), et à l'aide de l'outil SIG. L'image satellitaire utilisée a été mise en ligne le 29 Juin 2009 par la NASA. Elle a une résolution de 30m, et elle a été téléchargée depuis le site de la NASA (https://www.nasa.gov/topics/earth/features/20090629.html).

- La carte lithologique et la carte des degrés de résistance du sol ont été élaborées à partir de la carte géologique du Maroc au 1/1000000. Les substrats obtenus dans la carte des lithofaciès, ont été classé selon leur degré de résistance du sol en cinq classes. A chacune de ces classes on a affecté un code (a), (b), (c), (d) ou (e) selon le degré de friabilité comme préconisé dans les directives PAP/CAR (Tab1).

Tableau 1 : Classes des lithofaciès et leurs degrés de résistance

\begin{tabular}{|c|c|c|}
\hline \multicolumn{3}{|r|}{ Lithofaciès } \\
\hline Classe & Résistance & Type de matériel \\
\hline (a) & Très fort & $\begin{array}{l}\text { Roches compactes non altérées, conglomérats } \\
\text { fortement cimentés, etc. }\end{array}$ \\
\hline (b) & fort & Roche ou sol cohésif fracturé ou modérément altéré \\
\hline (c) & Moyen & $\begin{array}{l}\text { Roche ou sol sédimentaire faiblement ou } \\
\text { modérément compacté (ardoise, schiste, marne, etc.) }\end{array}$ \\
\hline (d) & Très faible & $\begin{array}{c}\text { Roches et /ou sols peu résistants ou } \\
\text { fortement/profondément altérés (marne, gypse, } \\
\text { ardoise argileuse, etc.) }\end{array}$ \\
\hline (e) & faible & $\begin{array}{c}\text { Sédiments ou sols meubles, non cohésifs et matériels } \\
\text { détritiques }\end{array}$ \\
\hline
\end{tabular}


- La carte d'érodibilité : La carte d'érodibilité a été élaborée par la superposition de la carte des pentes et de la carte de résistance des matériaux à 'érosion et la classification a été effectuée selon la matrice (Tab.2) préconisée par les directives PAP /CAR.

Tableau 2 : Matrice des pentes Vs friabilité

\begin{tabular}{|c|c|c|c|c|c|}
\hline \multirow{2}{*}{$\begin{array}{c}\text { Classes des } \\
\text { pentes }\end{array}$} & \multicolumn{5}{|c|}{ Classes des lithofaciès } \\
\cline { 2 - 6 } & $1(\mathrm{a})$ & $2(\mathrm{~b})$ & $3(\mathrm{c})$ & $4(\mathrm{~d})$ & $5(\mathrm{e})$ \\
\hline 1 & $1(\mathrm{EN})$ & $1(\mathrm{EN})$ & $1(\mathrm{EN})$ & $1(\mathrm{EN})$ & $2(\mathrm{~EB})$ \\
\hline 2 & $1(\mathrm{EN})$ & $1(\mathrm{EN})$ & $2(\mathrm{~EB})$ & $3(\mathrm{EM})$ & $3(\mathrm{EM})$ \\
\hline 3 & $2(\mathrm{~EB})$ & $2(\mathrm{~EB})$ & $3(\mathrm{EM})$ & $4(\mathrm{EA})$ & $4(\mathrm{EA})$ \\
\hline 4 & $3(\mathrm{EM})$ & $3(\mathrm{EM})$ & $4(\mathrm{EA})$ & $5(\mathrm{EX})$ & $5(\mathrm{EX})$ \\
\hline 5 & $4(\mathrm{EA})$ & $4(\mathrm{EA})$ & $5(\mathrm{EX})$ & $5(\mathrm{EX})$ & $5(\mathrm{EX})$ \\
\hline
\end{tabular}

- La carte d'occupation du sol a été élaborée à partir d'une image Landsat 8(Path 201, row 36) prise le 28/7/2017, qui a subi des traitements préalables ce qui a permis de synthétiser le NDVI (Normalized Difference Vegetation Index). L'interprétation visuelle de l'image résultante à base de la réflectance, a permis de cartographier l'activité chlorophyllienne d'une façon générale ce qui a permis de distinguer les terrains nus de ceux boisés. L'application d'une classification non supervisée, suivie d'une classification supervisée a permis d'avoir une idée sur la répartition des massifs forestiers et terrains nus en se basant sur les compositions colorées des différentes bandes de l'image. Une analyse comparative entre les cartes résultantes, les travaux réalisés dans la zone d'étude (HCEFLCD/DREFLCD-MA, 2004, 2007), les résultats de l'inventaire Forestier National (1990, actualisé en 2003) et les images «Google Earth», ont permis de se décider sur la répartition spatiale de l'occupation du sol dans le bassin versant étudié.

- L'estimation de la densité du couvert végétal et l'élaboration de sa carte ont été effectuées également sur la base du NDVI issu de la même image Landsat 8 sus-indiquée.

La carte de protection des sols a été produite par la superposition de la carte d'occupation du sol et de la carte de densité du couvert végétal. La carte résultante a été classée selon la matrice des directives PAP/CAR (Tab.3).

Tableau 3: Matrice d'occupation du sol Vs couvert végétal

\begin{tabular}{|c|c|c|c|c|}
\hline \multirow{2}{*}{ Occupation du sol } & \multicolumn{4}{|c|}{ Degré de couvert végétal } \\
\cline { 2 - 5 } & 1 & 2 & 3 & 4 \\
\hline 1 & $5(\mathrm{MB})$ & $5(\mathrm{MB})$ & $4(\mathrm{~B})$ & $4(\mathrm{~B})$ \\
\hline 2 & $5(\mathrm{MB})$ & $5(\mathrm{MB})$ & $4(\mathrm{~B})$ & $3(\mathrm{~A})$ \\
\hline 3 & $3(\mathrm{M})$ & $2(\mathrm{~A})$ & $1(\mathrm{MA})$ & $1(\mathrm{MA})$ \\
\hline
\end{tabular}


- La carte des états érosifs résulte de la superposition de la carte d'érodibilité et de la carte de protection du sol, elle constitue la 7 ème et la dernière étape de la phase prédictive et donne une idée géo-localisée sur le potentiel et les tendances générales de l'érosion.

\section{La Phase Descriptive}

L'approche descriptive se fait en une seule étape. Elle consiste à faire une évaluation qualitative en décrivant les formes et les processus d'érosion réellement existant dans la zone d'étude. Dans cette phase on distingue d'abord deux grandes catégories de milieux géographiques: les milieux stables, non affectés par l'érosion et les milieux instables, affectés par divers processus d'érosion en indiquant leur indice de dominance. Cette carte a été réalisée par des observations sur le terrain, par exploitation des données existantes (documents d'aménagement des bassins versant et d'aménagement forestier, études relatives à l'érosion et autres) et par exploitation des images "Google Earth », tout en se référant à la carte prédictive des états érosifs comme canevas cartographique et thématique.

\section{La Phase d'intégration et de Consolidation}

La phase d'intégration est la neuvième étape de la procédure PAP/CAR, et comme son nom l'indique, elle consiste à intégrer les résultats des huit étapes précédentes obtenus dans les deux premières phases (prédictive et descriptive).Le résultat final qui est la carte consolidée d'érosion PAP/CAR est donc une carte qualitative obtenue par la superposition des informations qualitatives issues des phases prédictive et descriptive.

L'approche PAP/CAR a été résumée dans le schéma méthodologique (Fig.2) suivant :

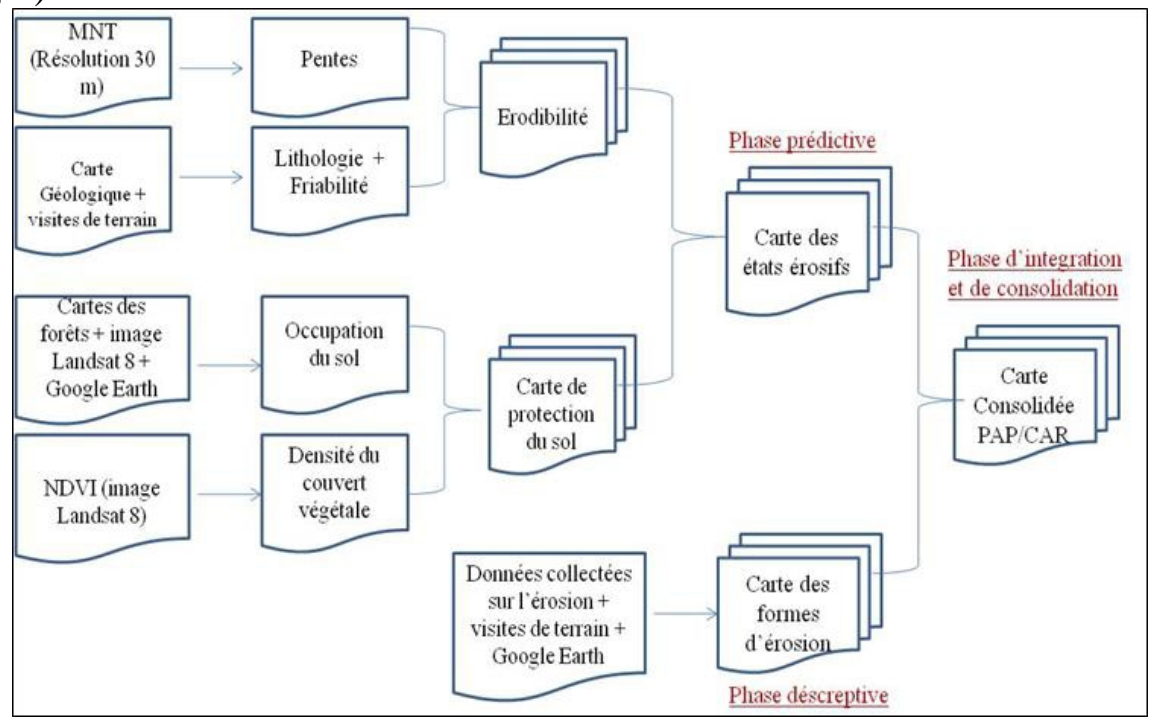

Figure 2: Schéma méthodologique de l'approche PAP/CAR 
La réalisation de la méthode PAP/CAR fait appel à un ensemble de données qu'il faut collecter auparavant. Ces données sont liées à la topographie, à la géologie et au couvert végétal. L'exploitation des données existantes (relatives à l'érosion dans la zone d'étude) et les visites d'observations de terrain sont d'une grande importance.

Les images satellitaires de type Landsat 8 et Google Earth et le modèle numérique de terrain (MNT) provenant d'images satellitaire ASTER GDEM de $30 \mathrm{~m}$ de résolution ainsi que les logiciels dédiés à la télédétection et au (SIG) ont été utilisé dans ce travail.

\section{Résultats et Discussion}

\section{Approche Prédictive}

La phase prédictive est composée de 7 étapes de traitement de données qui aboutissent dans leur dernière étape à la cartographie des unités homogènes des états érosifs, fournissant le canevas pour la cartographie du potentiel et des tendances générales de l'érosion hydrique du sol.

\section{Carte des Pentes}

L'analyse de la carte des pentes (Fig.3) et de la table attributaire qui lui est relative montre que les classes des fortes (20-35\%) à très fortes (35\%< ) occupent $36 \%$ du bassin, et elles se sont plus concentrée dans les parties aval du bassin correspondantes au plateau central. On trouve aussi ces classes de pente sur les rives de l'Oued Beht, sur les falaises et aux points où prennent naissance les cours d'eau. La classe de pente modérée (3-12\%) occupe à elle seule la grande partie (37\%) et la classe de pente moyenne (12-20\%) occupe $19 \%$ tandis que la classe à faible pente $(0-3 \%)$ n'occupe que $(08 \%)$ de la surface du bassin. Les pentes faibles et modérées occupent surtout la partie amont et le centre du bassin de Beht. 


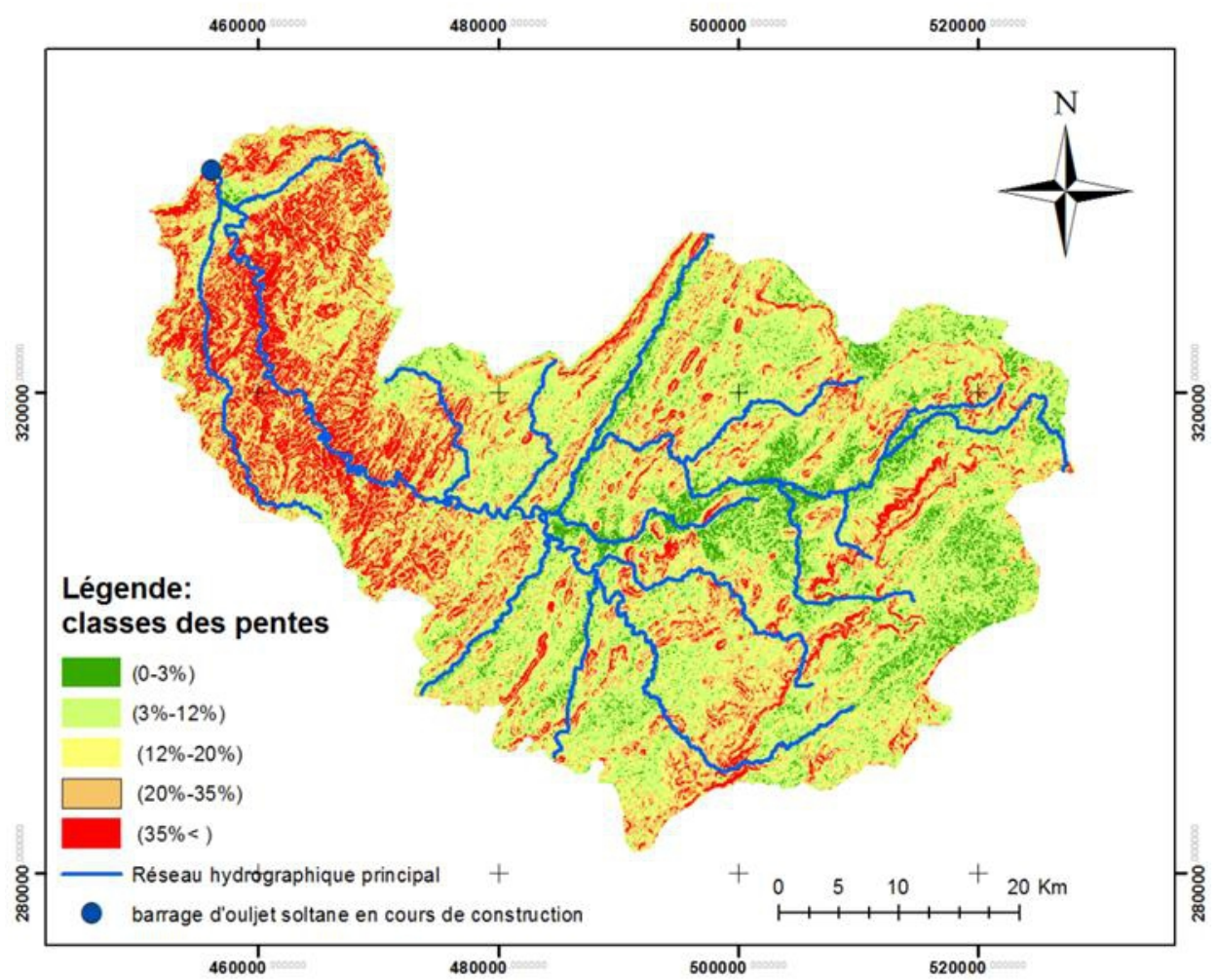

Figure 3: Carte des pentes du bassin versant de l'Oued Beht en amont du barrage d'Ouljet Soltane

\section{Carte Lithologique et Carte de Friabilité}

La carte lithofaciès du bassin versant de l'Oued Beht (Fig. 4) montre que ce bassin est dominé par les Schistes qui se trouvent en mélange avec des grès, des calcaires ou des basaltes. Cette classe s'étend sur $77 \%$ de la surface du bassin et correspond au meseta centrale, suivie parla classe des basaltes et ankaratrites plio-quaternaires qui occupent $(12 \%)$ et se situe dans la partie amont du bassin d'étude surtout dans la région d'Azrou et Timahdite. La classe des terrasses alluviales ou fluviatiles occupe près de $6 \%$, et la classe de conglomérat, grés et argile occupe $4 \%$ du bassin. Des passages de calcaires saillants ou corniches occupent près de $1 \%$ du bassin. 


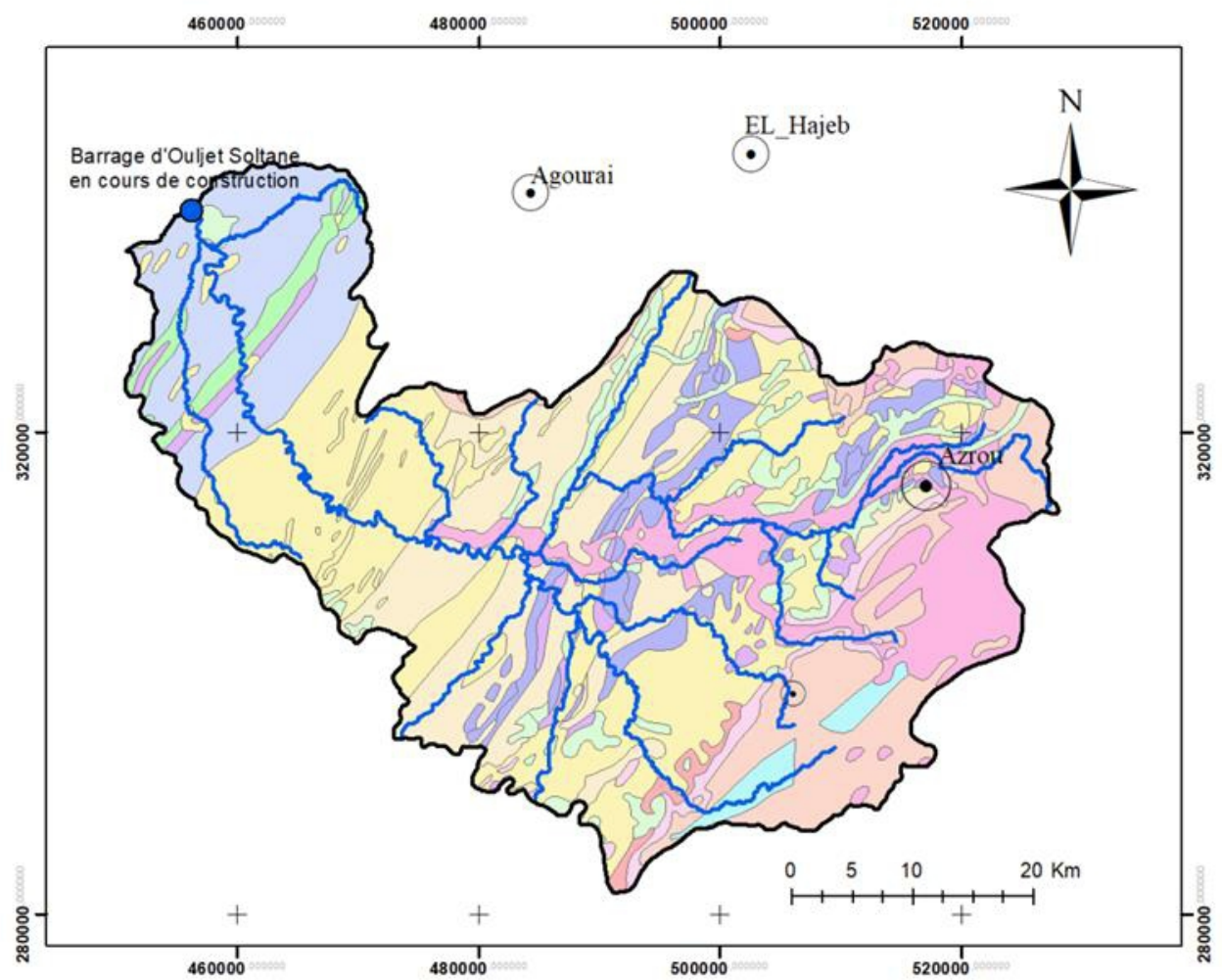

Légende

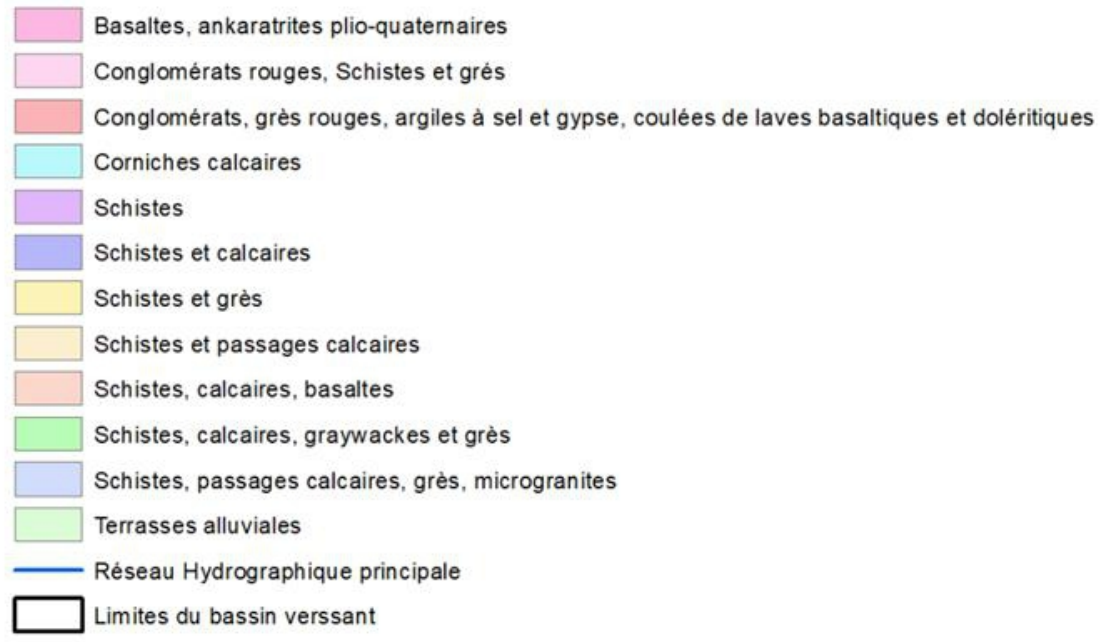

Figure 4: Carte géologique du bassin versant de l'oued Beht en amont du barrage d'Ouljet Soltane (Carte géologique du Maroc (1/1M), maquette achevée en 1977, révisée en 1982 et publiée en 1985) 
L'analyse de la carte (Fig.5) relative au degré de résistance du sol à l'érosion montre que les classes de degré de résistance faible (d) à très faible (e) occupent $20 \%$ du bassin et que la classe à moyen résistance (c) occupe 34 $\%$ de la superficie alors que les classes de résistance forte (b) à très forte (a) représente $44 \%$ de la surface de ce bassin versant.

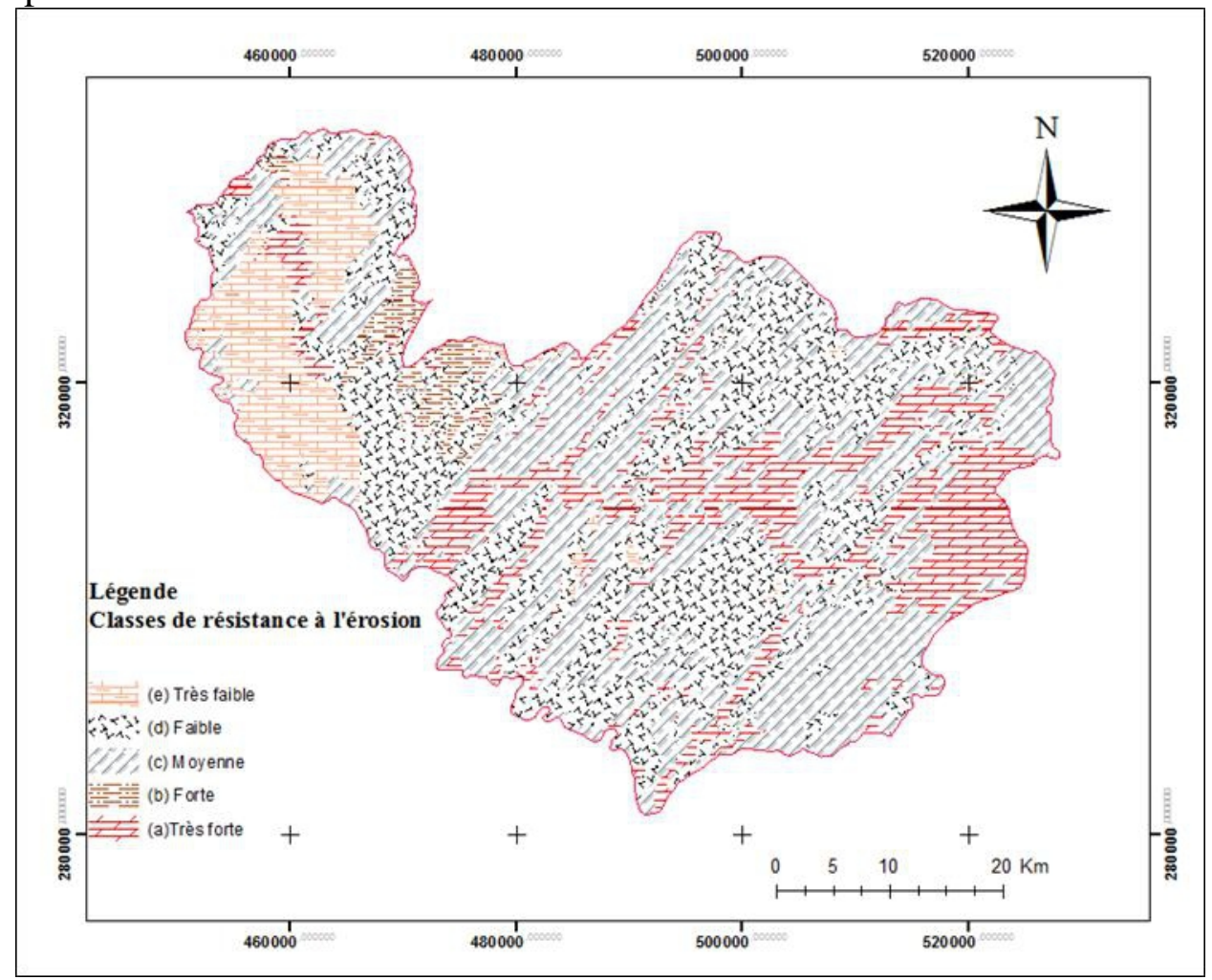

Figure 5: Carte du degré de résistance du sol à l'érosion dans le bassin versant de Beht en amont du barrage d'Ouljet Soltane

\section{Carte d'érodibilité}

L'analyse de la carte d'érodibilité (Fig.6) et de la table attributaire qui lui est relative (Fig.7) montre que les classes de degré d'érodibilité forte (EA) à extrême (EX) occupent $83 \%$ du territoire du bassin d'étude tandis que les classes d'érodibilité faible (EN) à modérée (EB) n'occupent que $9 \%$ de la surface de ce bassin versant. La dominance des terrains d'érodibilité forte à extrême, est due à la combinaison des terrains de faible résistance constitués d'éléments friables (sols argilo-schisteux, sol sédimentaire non cohésifs, etc..) et de forte pentes qui augmentent la force d'inertie des pluies et leurs accélérations ainsi que la force locomotrice des eaux de surface d'une façon générale. 


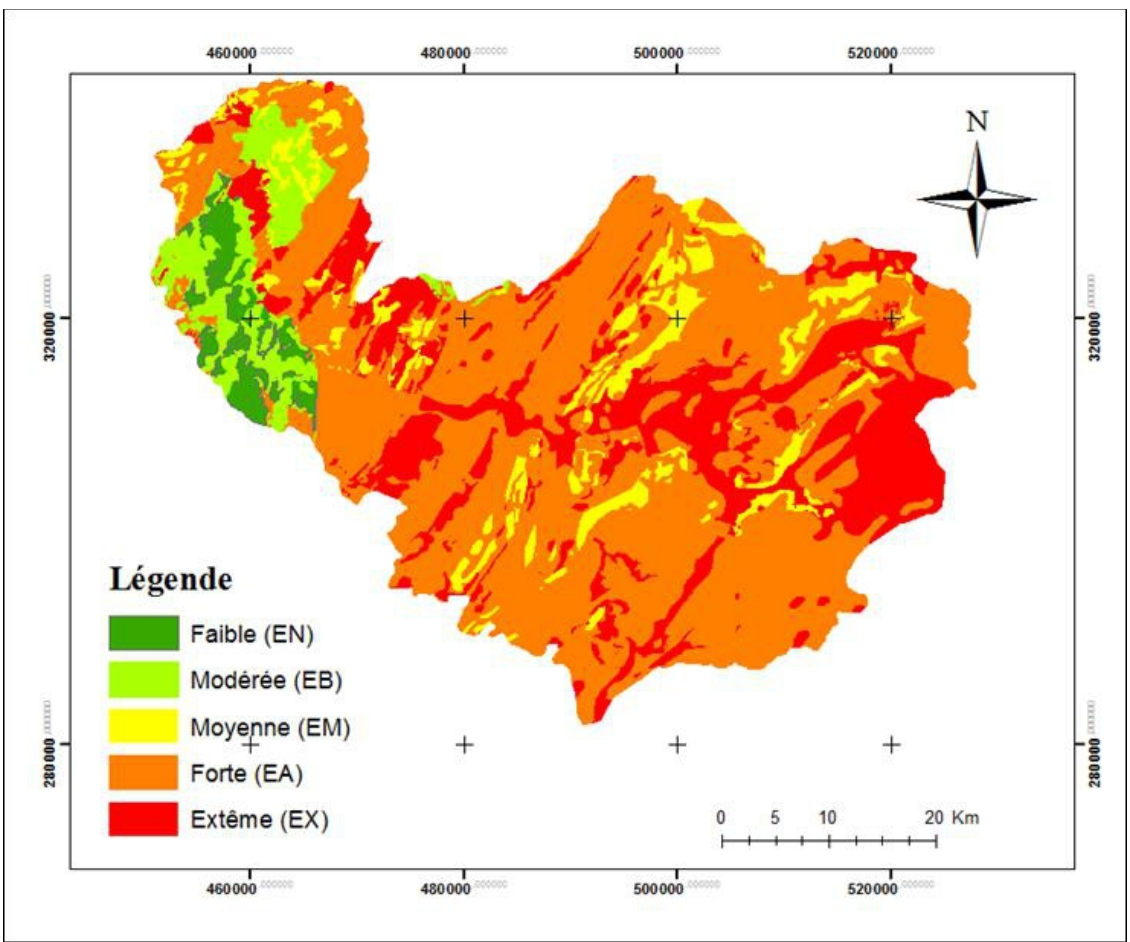

Figure 6: Carte d'érodibilité dans le bassin versant de l'oued Beht en amont du barrage d'Ouljet Soltane

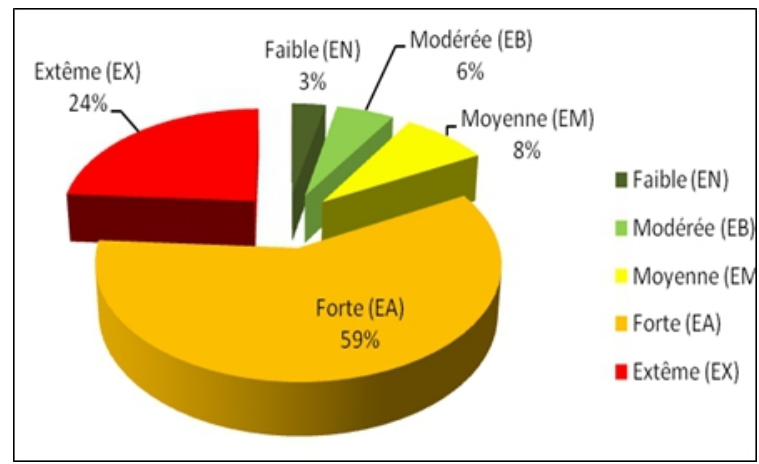

Figure 7: Répartition des classes d'érodibilité des sols dans le bassin versant de l'oued Beht en amont du barrage d'Ouljet Soltane

\section{Carte d'occupation du Sol}

Les thèmes identifiés sur la carte d'occupation du sol (Fig.8) sont les forêts, les matorrals, les terrains arboricoles, les terrains agricoles, les terrains de parcours, les sols nus ou dénudés, les plans ou cours d'eau et les constructions. L'analyse de cette carte permet de constater que les terrains de parcours destinés au pâturage traditionnel des troupeaux ( bovins et surtout ovins et caprins) sont majoritaires en matière de surface occupée (44\%), les forêts et les matorrals représentent $33 \%$ du couvert du bassin, les terrains 
agricoles et arboricoles occupent $10,7 \%$ et les terrains nus ou dénudés occupent $12 \%$, les constructions et les eaux ne dépassent pas $0,3 \%$ et peuvent être négligés (Fig.9).

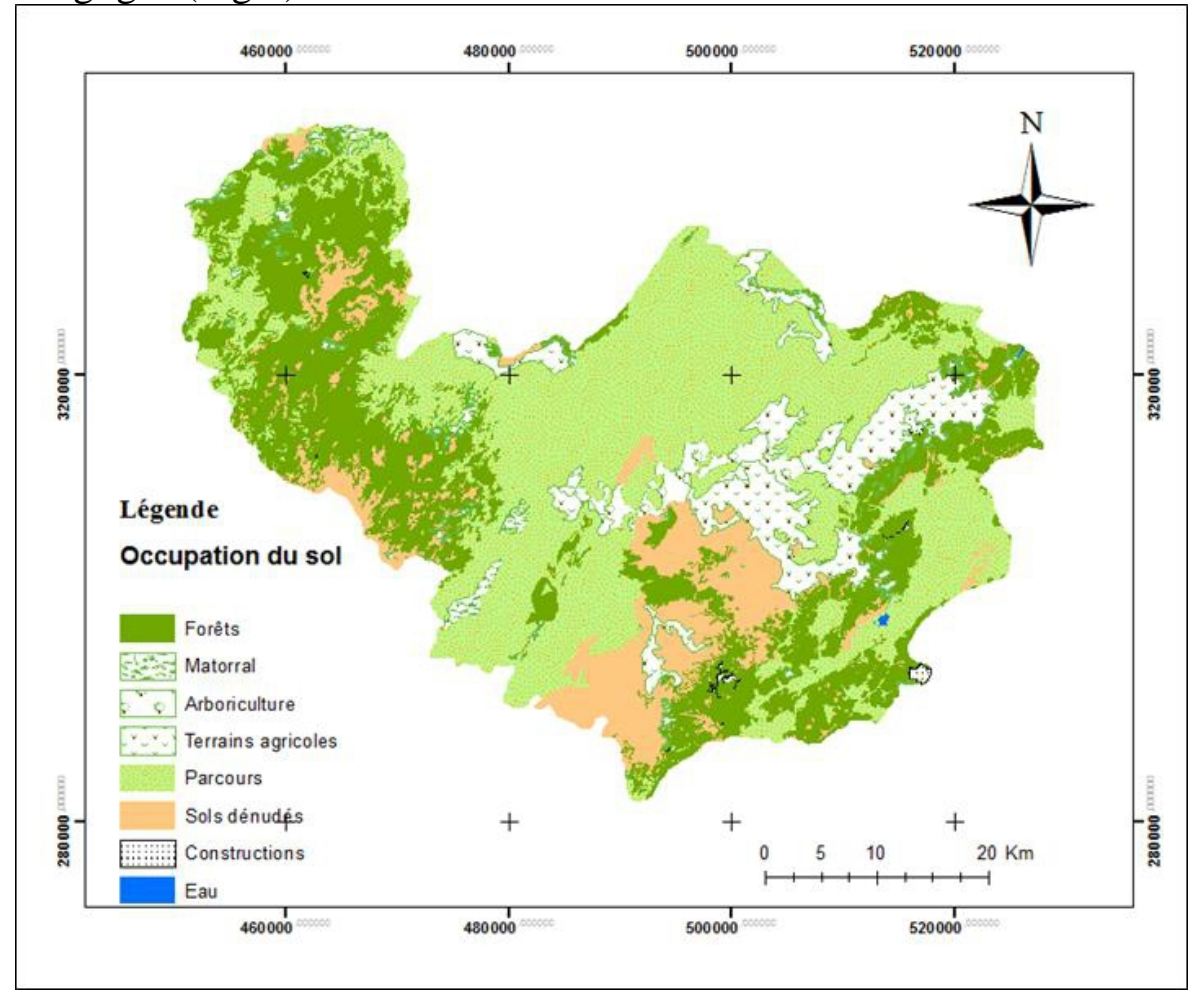

Figure 8: Carte d'occupation du sol dans le bassin versant de Beht en amont du barrage d'Ouljet Soltane

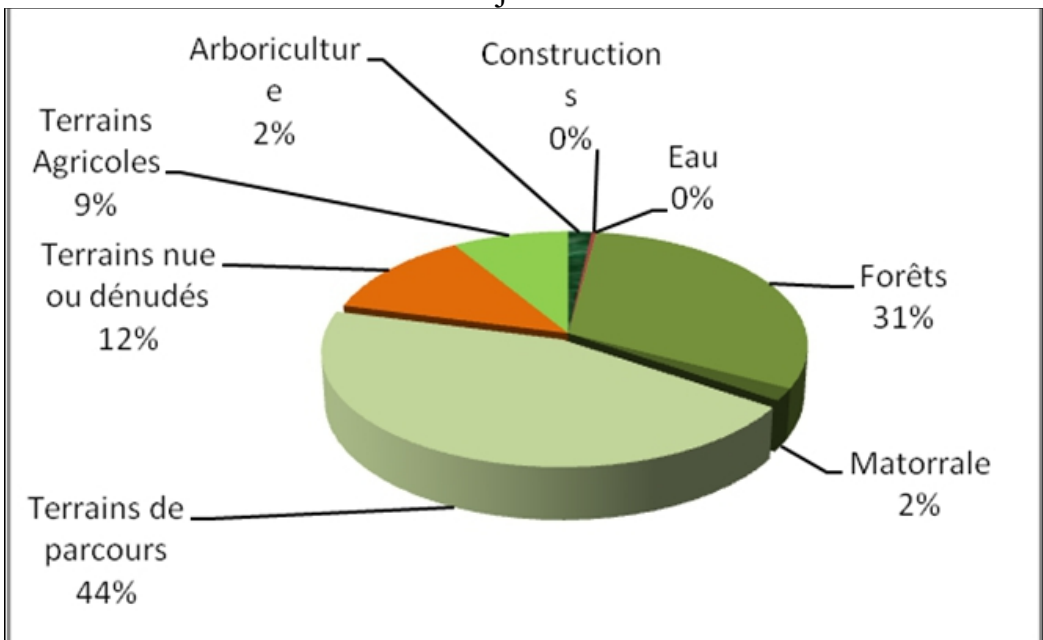

Figure 9: Répartition des classes d'occupation du sol dans le bassin versant de Beht en amont du barrage d'Ouljet Soltane 


\section{Carte de Densité du Couvert Végétal}

La couverture végétale joue un rôle important dans la protection du sol contre l'érosion du fait qu'elle brise la force érosive des eaux de surface et favorise l'infiltration des eaux aux horizons profondes du sol; mais cette protection est sans doute liée au degré de densité du couvert végétal.

La carte du degré de densité du couvert végétal (Fig.10) a permis d'avoir quatre classes représentant différents degré du couvert : très élevée $(75 \%<)$, élevée $(50-75 \%)$, moyenne $(25-50 \%)$ et faible $(<25 \%)$, en plus de la classe à sol nu ou dénudé.

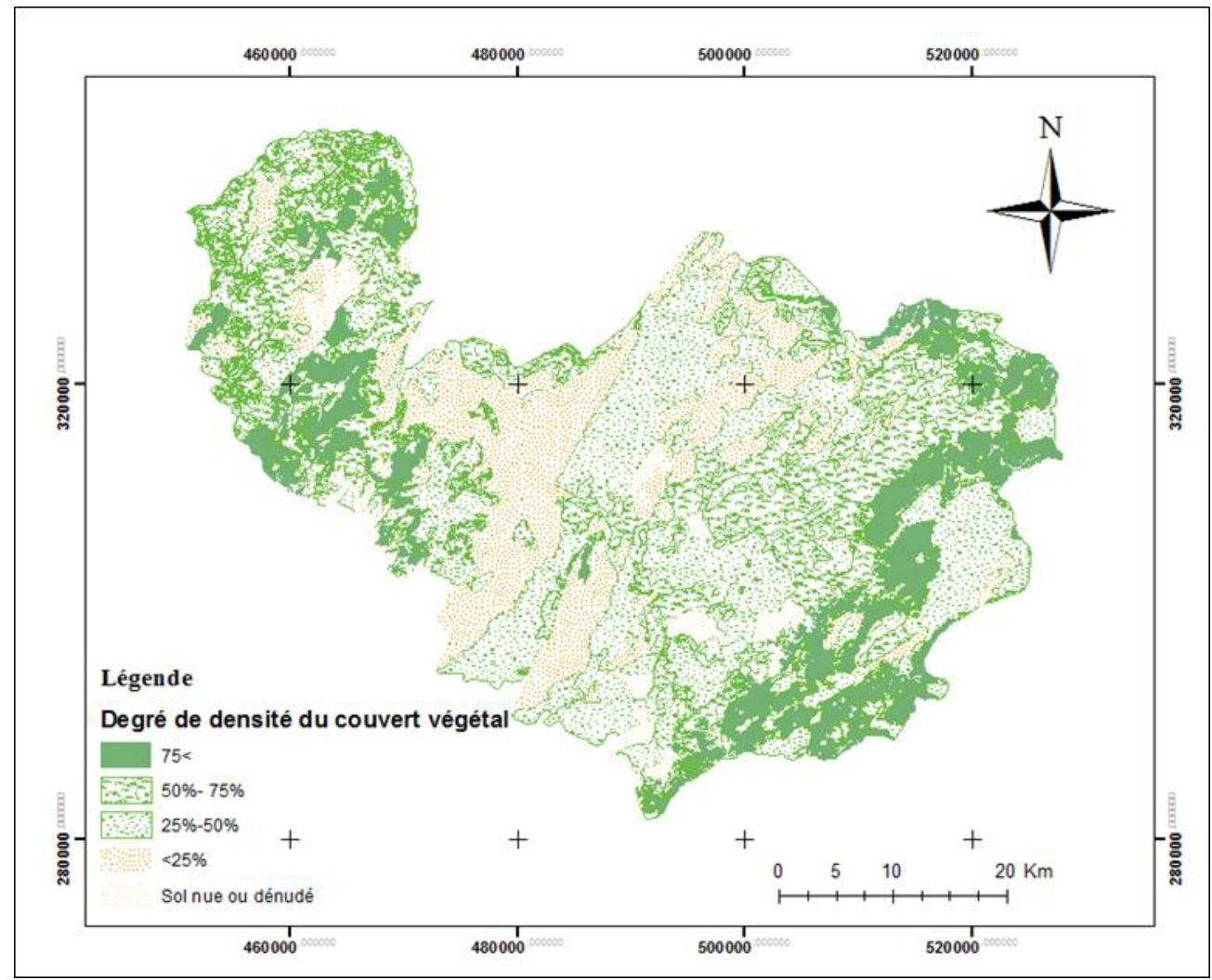

Figure 10: Carte de la densité du couvert végétal dans le bassin versant de Beht en amont du barrage d'Ouljet Soltane

L'étude de la carte de densité du couvert végétal montre que la classe de densité moyenne occupe 39\% de l'aire d'étude et que la classe à densité élevée à très élevée occupe $37 \%$ du bassin versant alors que la classe dont la densité du couvert végétal est faible ou nulle occupe $24 \%$. On peut dire que notre bassin d'étude possède une bonne densité du couvert végétal comparativement à d'autres bassins versant avoisinant (Fig. 10 et 11). 


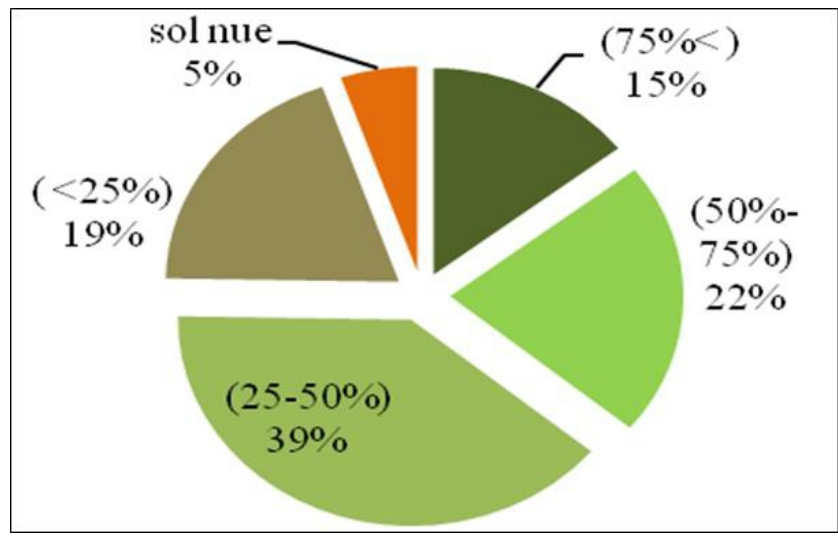

Figure 11: Répartition de la densité du couvert végétal dans le bassin versant de Beht en amont du barrage d'Ouljet Soltane

\section{Carte de Protection des Sols}

La carte de protection du sol (Fig.12) a permis d'avoir cinq classes du degré de protection du sol: très faible $(\mathrm{MB})$, faible $(\mathrm{B})$, moyenne $(\mathrm{M})$, élevée (A) et très élevée (MA).

L'analyse de la carte de protection du sol permet de relever (Fig.13) que les classes de faible (B) à très faible protection du sol (MB) occupent la moitié de la superficie du bassin (50\%). Cela montre qu'une partie importante des sols est faiblement protégée contre l'érosion ce qui peut être expliqué par l'importance des terrains de parcours (44\%) ajoutés aux terrains nus ou dénudés (12\%). Les classes dont la protection du sol est considérée élevée à très élevée couvrent $38 \%$ de la superficie du bassin. 


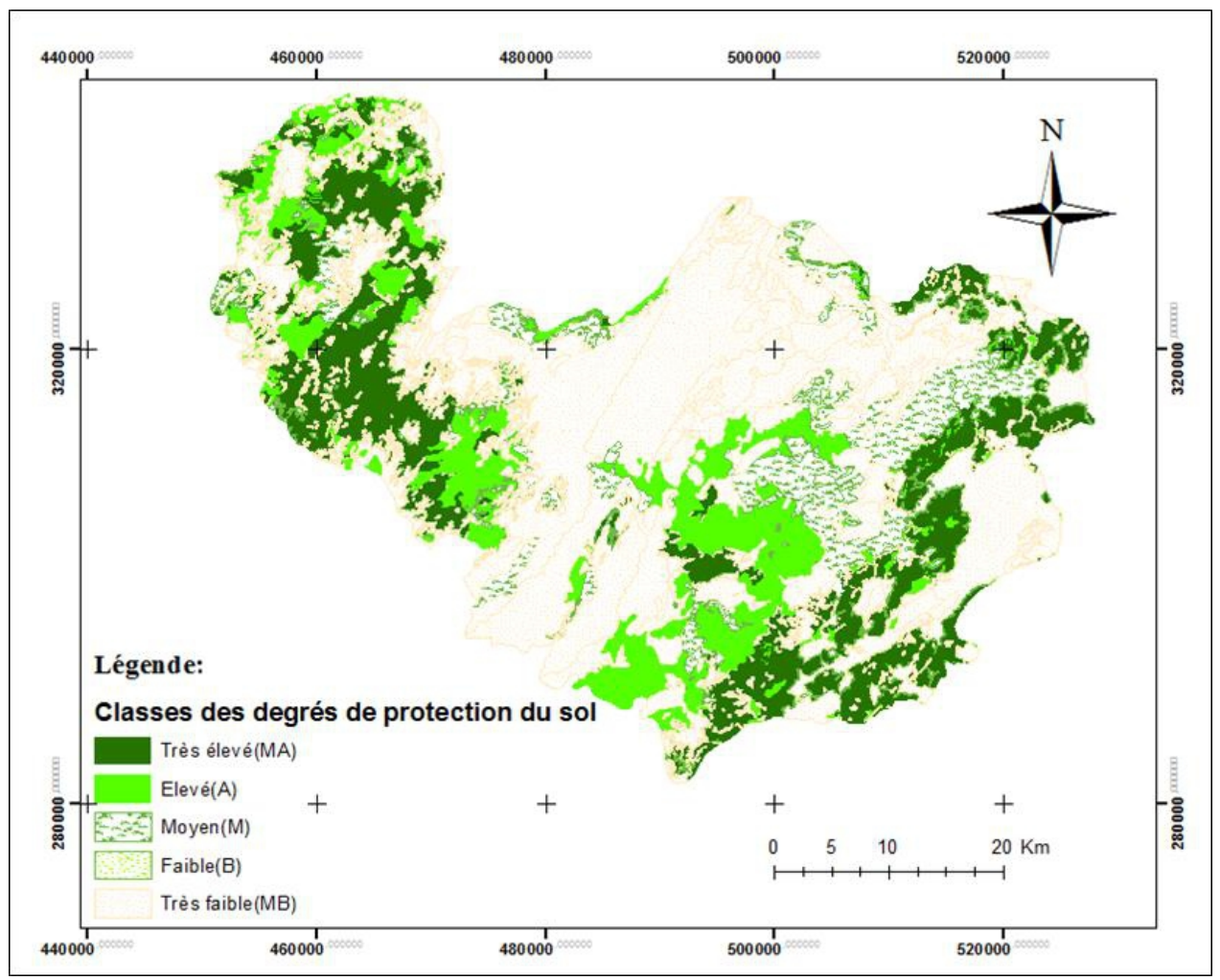

Figure 12: Carte de protection du sol dans le bassin versant de Beht en amont du barrage d'Ouljet Soltane

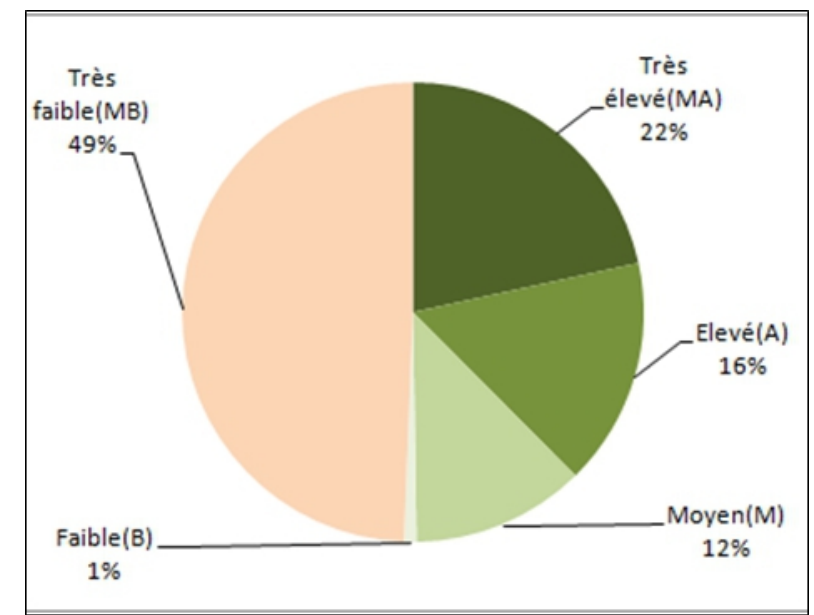

Figure13: Répartition des classes de protection du sol dans le bassin versant de Beht en amont du barrage d'Ouljet Soltane 


\section{Carte des États Érosifs}

La carte des états érosifs résultante est présentée en cinq classes reflétant différents degrés d'érosion. Il s'agit des classes de degré très faible, faible, notable, élevé et très élevé (Fig.14).

L'analyse des données de cette carte montre que les classes de risque d'érosion élevé à très élevé occupent la majorité du territoire de ce bassin versant (59\%), et que seulement $26 \%$ de la superficie de ce bassin présentent un risque à l'érosion de degré faible à très faible. Les parties à haut risque d'érosion dominent essentiellement le centre et les parties aval du bassin et correspondent à la Meseta centrale surtout à sol friable et sur les fortes pentes comme le cas des rives de l'oued Beht (Fig.15).

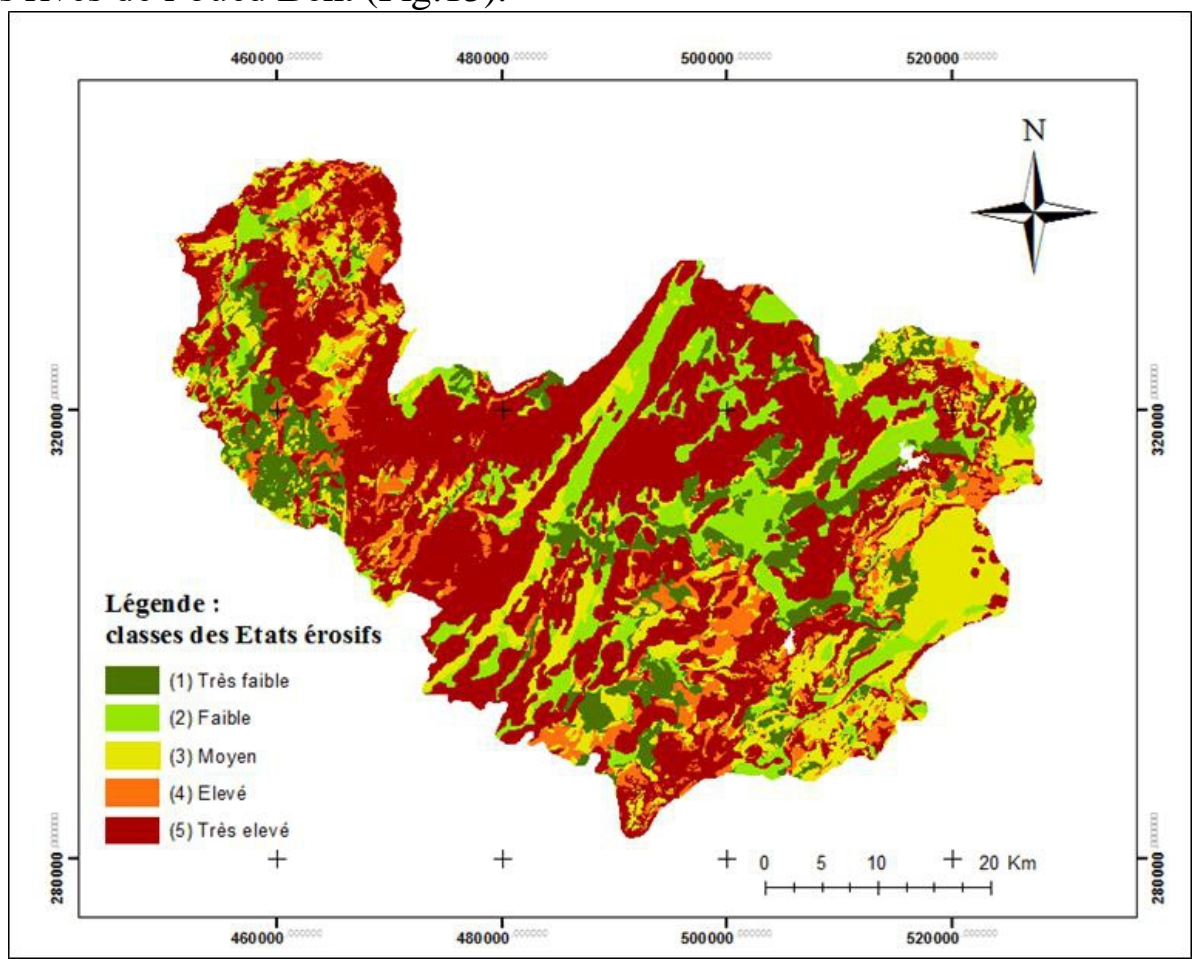

Figure 14: Carte des états érosifs dans le bassin versant de Beht en amont du barrage d'Ouljet Soltane 


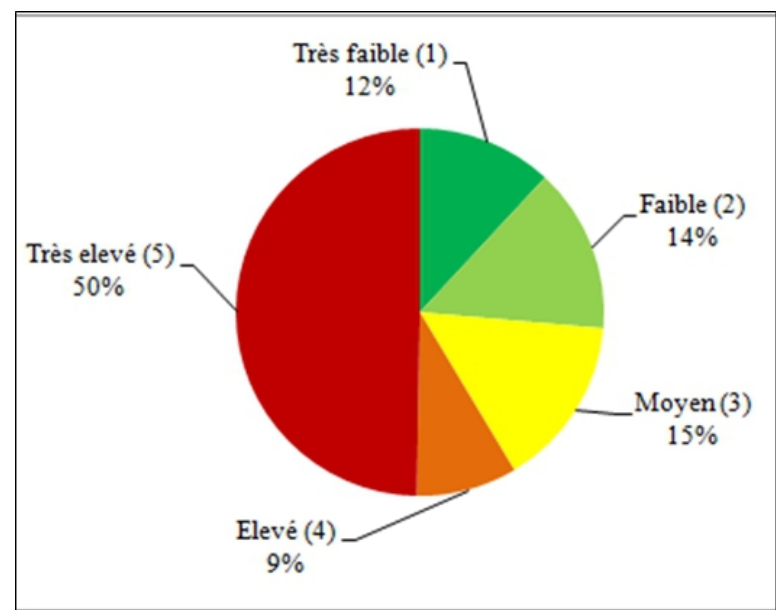

Figure 15: Répartition des classes des états érosifs dans le bassin versant de Beht en amont du barrage d'Ouljet Soltane

\section{Phase Descriptive (Formes d'érosion)}

La carte des formes d'érosion résultante (Fig.16) montre que ce bassin versant englobe toutes les formes d'érosion et avec des degrés d'abondance différentes. Ces formes d'érosion se trouvent généralement combinées sur l'ensemble du territoire du bassin, mais en se basant sur l'approche abondancedominance on arrive à attribuer à chaque aire géographique la / ou les formes d'érosion qui y dominent.

L'analyse des données (Fig. 16 et 17) permet de déduire que les milieux considérés comme stables ou stabilisés par des plantations végétales avec un faible risque d'instabilité n'occupent que $21 \%$ de l'aire d'étude, alors que les milieux instables occupent le reste (79\%). L'érosion dans ces milieux se manifeste par différentes formes: la classe où se combinent à la fois l'érosion en nappe, l'érosion linéaire et l'érosion ravinante occupe près de $36 \%$ du bassin d'étude et la classe combinée par l'érosion en nappe et l'érosion linéaire localisée à dominante couvre $29 \%$ du bassin. 


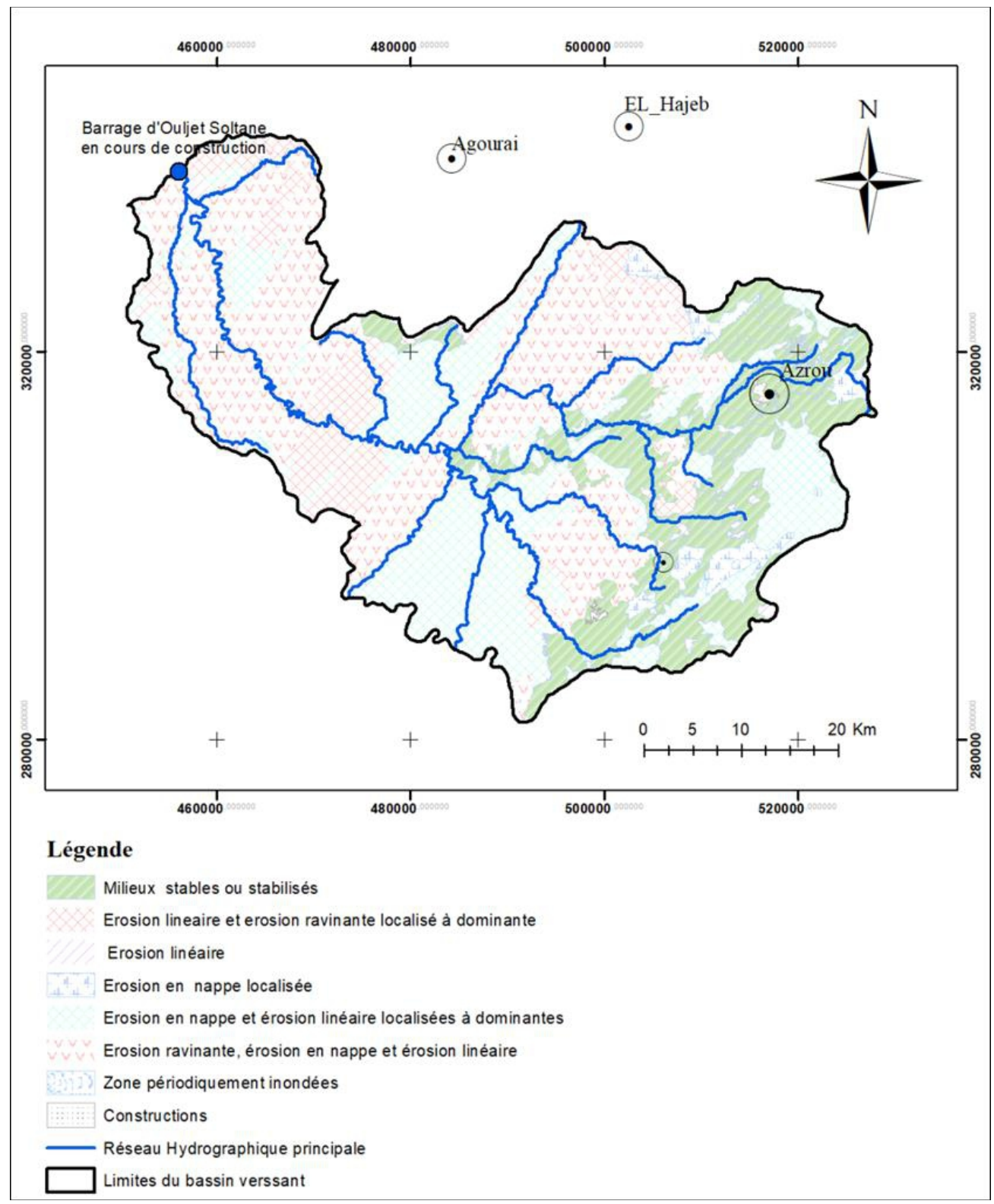

Figure 16: Carte des formes d'érosion dans le bassin versant de Beht en amont du barrage d'Ouljet Soltane 


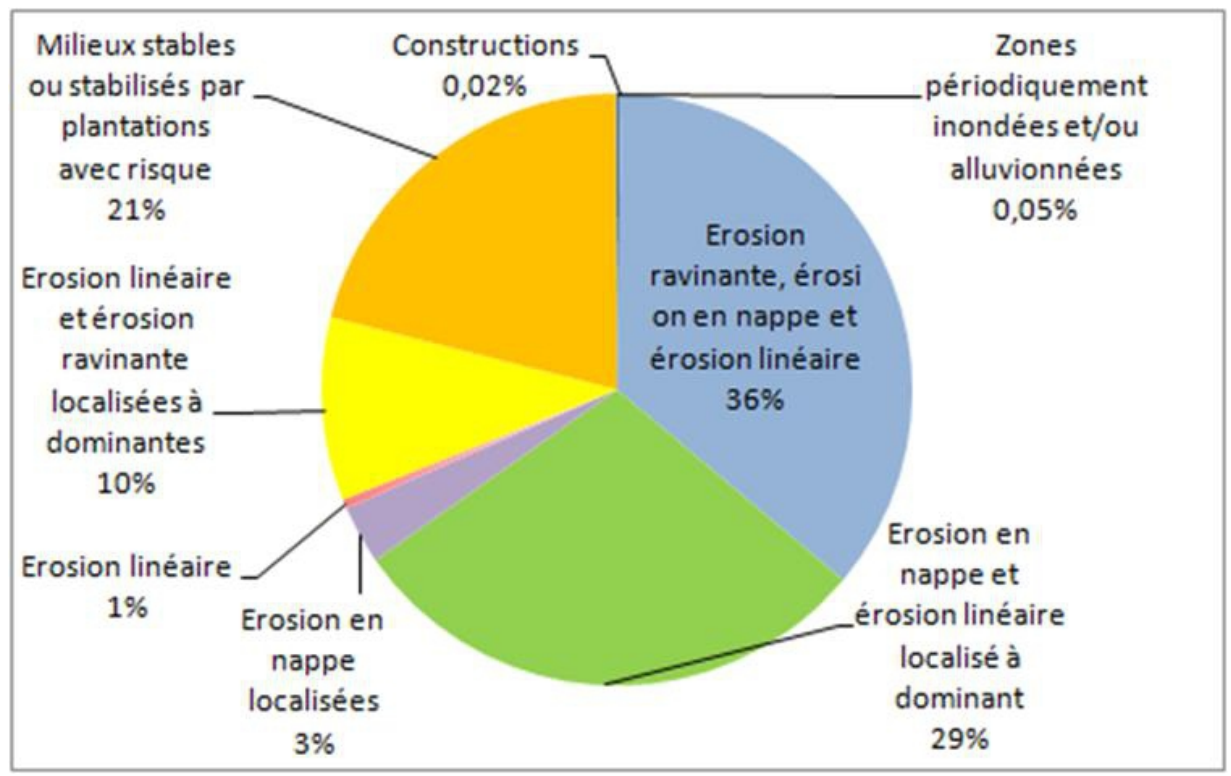

Figure 17: Répartitions des formes d'érosion dans le bassin versant de Beht en amont du barrage d'Ouljet Soltane (observation personnelle)

\section{Phase d'intégration et de Consolidation}

L'analyse de la carte consolidée d'érosion permet de déduire que:

$>$ Le bassin versant de Beht en amont du barrage d'Ouljet Soltane, englobe toutes les formes d'érosion qui se trouvent éparpillées sur tout le territoire du bassin avec des degrés d'abondance différents et avec une dominance relative de l'érosion en nappe, de l'érosion linéaire et de l'érosion en ravins. Cela est en accord avec le fait que les milieux stables occupent seulement $21 \%$ du bassin.

$>$ Les classes de risque d'érosion élevé à très élevé occupent (59\%) du territoire de ce bassin versant et coïncident avec la majeur partie de la classe où se combinent à la fois l'érosion en nappe, l'érosion linéaire et l'érosion en ravin. Des formes individualisées de ravins avec des creusements profonds sont observés surtout au niveau des principaux cours d'eau sur les sols à faible résistance des matériaux. Les classes d'érosion linéaire et d'érosion en nappe existent aussi, mais avec un degré relativement faible.

$>$ Les zones d'érosion faible à modérée se superposent en grande partie avec l'érosion en nappe dans sa forme localisée ou parfois combinée avec l'érosion linéaire, notamment au bord des affluents de l'oued Beht. On remarque aussi que des zones faisant partie des classes de faible à très faible degré d'érosion contiennent parfois la forme d'érosion en ravin surtout localisée, cela peut être expliqué par la combinaison à la fois d'un sol friable à base de schiste et de grés et de la faible protection 
du sol (zones correspondant dans la plus part aux terrains de parcours ou à faible densité végétale).

> Les milieux considérés comme stables ou stabilisés présentent un risque d'instabilité qu'il ne faut pas négliger. Ces milieux correspondent aux terrains qui ont présenté auparavant des indices d'érosion et qui ont bénéficié de mesures de lutte contre l'érosion sous forme de plantations forestières pour les terrains appartenant au domaine forestier ou à base d'arbres fruitière (arboriculture) pour les terrains privés via des incitations initiés par le HCEFLCD (dans le cadre des contrats de défense et restauration du sol (DRS). Le problème se pose pour les terrains de parcours qui se trouvent souvent soumis au régime collectif et qui subissent rarement des corrections contre l'érosion vu leur statut juridique compliqué. Dans ces milieux l'érosion hydrique du sol est nettement active, et elle se manifeste sous différentes formes et à différents degré.

L'érosion linéaire et l'érosion ravinante occupent près de 36\% du bassin d'étude, et la classe combinée de l'érosion en nappe et de l'érosion linéaire localisée à dominante, couvre $29 \%$ du bassin versant étudié. 


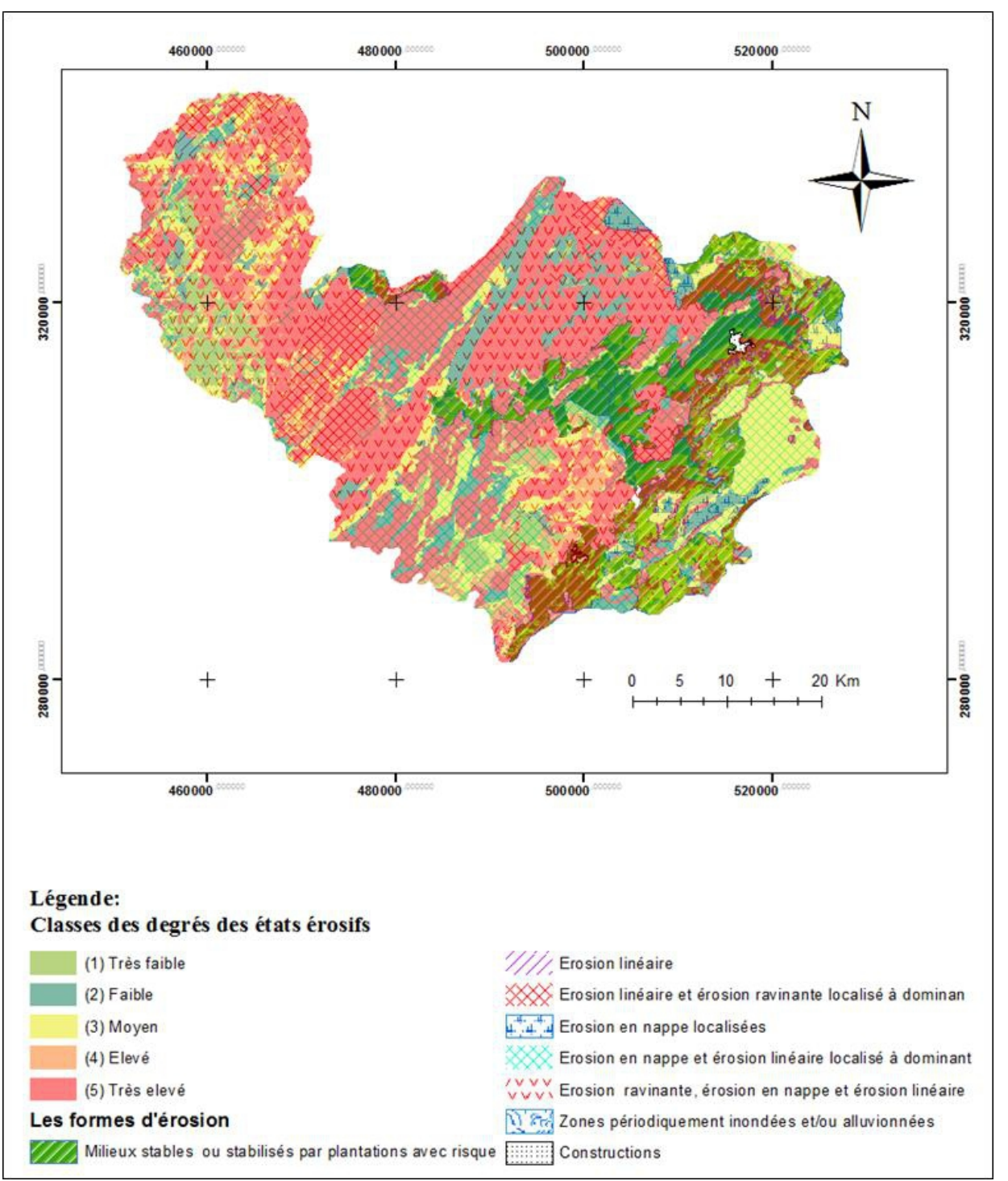

Figure 18: Carte consolidée PAP/CAR de l'érosion dans le bassin versant de Beht en amont du barrage d'Ouljet Soltane

\section{Discussion}

Les résultats obtenus dans cette étude qualitative de l'érosion par la méthode $\mathrm{PAP} / \mathrm{CAR}$ s'avère satisfaisante comparativement à d'autres études sur l'érosion effectuées dans le bassin de l'Oued Beht en général (Mimich et al, 2018; Lakhili et al, 2017; Gaatib et al, 2015 et HCEFLCD/DREFLCD-MA, 2007).Ces études ont tous confirmé que ce bassin est assujetti à une érosion forte manifesté par toutes les formes d'érosion et surtout l'érosion ravinant, l'érosion en nappe, et l'érosion linéaire. D'autre études similaires ont été menées sur des bassins versants avoisinants ont utilisé la méthode PAP/CAR 
(Ousmana et al, 2017; Ouallali et al, 2016 et Mesrar, 2016), et ont trouvé des résultats qui confirment l'utilité de la méthode utilisée et l'ampleur de l'érosion dans ces zones d'étude. En revanche il faut noter que cette méthode a été critiquée par d'autres chercheurs vue les limites qu'elle présente. En effet la méthode PAP/CAR n'intègre pas le facteur climatique qui réside dans l'agressivité des pluies constituant le facteur locomoteur qui désarticule et mobilise les sols érodés. Aussi, en ce qui concerne le facteur topographique, la méthode PAP/CAR ne tient compte que du facteur pente et néglige le facteur longueur de la pente contrairement à la méthode RUSLE qui intègre les deux facteurs dan une équation bien définit. L'importance de ce facteur réside dans le fait que la force érosive des eaux de pluie augmente proportionnellement avec l'énergie cinétique des eaux qui est influencé par la longueur de la pente. D'autre part la méthode PAP/CAR intègre dans sa partie descriptive le facteur lié aux formes d'érosion existantes sur le milieu, ce facteur que je trouve très important donne une visibilité claire sur l'état du milieu et les tendance futures des formes d'érosion et permet de définir les outils de lutte contre ce fléaux, ce qui ne l'est pas dans la méthode RUSLE.

La méthode PAP /CAR, comme autres méthodes d'études de l'érosion, n'est pas idéale, mais c'est une méthode complémentaire visant à mieux comprendre le phénomène de l'érosion et ses processus en vue de définir les outils efficace de lutter contre.

\section{Conclusion}

L'étude de l'érosion hydrique du sol dans le bassin versant de Beht en amont du barrage d'Ouljat Soltan, par application de la méthode qualitative PAP /CAR, a intégré plusieurs facteurs causaux (pente, lithologie, occupation des sols, densité du couvert végétal et formes d'érosion réellement existant). Le résultat de cette étude montre que ce bassin versant est exposé à une érosion active et apparente sur près des $3 / 4$ de la superficie du bassin, et que plus de la moitié de la zone d'étude présente une forte à très forte érosion. Elle montre aussi que l'érosion se manifeste par toutes ses formes et notamment la classe ou se combinent à la fois l'érosion en nappe, l'érosion linéaire et l'érosion ravinante $(36 \%)$. La classe combinée par l'érosion en nappe et l'érosion linéaire localisées à dominantes est en second lieu et occupe (29\%). Ces zones à érosion active correspondent généralement aux terrains accidentés avec un taux de friabilité des sols assez élevé et une densité du couvert végétal faible à très faible. Le ravinement est fortement observé sur les parties centrales et avals $\mathrm{du}$ bassin versant qui correspondent au Meseta centrale où le réseau devient de plus en plus ramifié et individualisé en allant vers l'aval.

Les zones les plus touchées par l'érosion se trouvent répartis en aval et au centre du bassin et correspondent aux terrains à fortes pentes, tandis que les moins érodées se localisent au niveau des terrains à faible pente (terrains 
agricoles et dépressions). Les milieux considérés comme stables ou stabilisés présentent un risque d'instabilité important par endroit, et doivent être sous contrôle.

On peut dire que: la modélisation de l'érosion hydrique du sol par méthode PAP/CAR, bien qu'elle soit relativement ancienne (1998), mais d'une part avec l'avancement sans cesse des techniques de la Géomatique (SIG et télédétection) surtout au niveau de la performance des SIG et la résolution de plus en plus amélioré des images satellitaires et d'autre part l'accroissement du degré d'érosion par les changements climatiques, ont repoussé les recherches sur ce phénomène, et cette méthodes est parmi celles qui ont donné des résultats satisfaisants et approuvés par plusieurs chercheurs.

\section{References:}

1. Beaudet, G. (1969). Le plateau central marocain et ses bordures: étude géomorphologique. (Service geologique du Maroc, Ed.). Service geologique $\mathrm{du}$

Maroc. https://scholar.google.com/scholar?hl=fr\&as_sdt=0\%2C5\&q=Beaude $\mathrm{t}+\mathrm{G} . \% 2 \mathrm{C}+1969$.+Le+plateau+central+marocain+et+ses+bordures $\% 3$ A+tude+gomorphologique.+Inframar\%2C+Rabat+.478p\&btnG $=$

2. Celik, I., Aydin, M., \& Yazici, U. (1996). "A review of the erosion control studies during the republic period in Turkey. In 1st International Conference on land degradation, Adana, Turkey (pp. 1014).

3. Gaatib, R. El, Larabi, A., \& Faouzi, M. (2015). Integrated elaboration of priority planning of vulnerable areas to soil erosion hazard using Remote Sensing and GIS techniques: A pilot case of the Oued Beht Watershed ( Morocco ). J. Mater. Environ. Sci., 6(11), 3110-3126.

4. Haitami, M. (2017). PROBLÉMATIQUE DE L ' EAU AU MAROC L ' heure est à 1 ' action. Le Matin, Maroc, pp. 1-12. Retrieved from https://epaper.lematin.ma/lematin/special/eau-au maroc/2017/10/16/.../page-5.html

5. HCEFLCD/DREFLCD-MA. (2004). Etudes d'aménagement concerte des forêts et des parcours collectifs de la province d'Ifrane: carte d'occupation des sols, rapport $\mathrm{n}^{\circ} 2$, Parc National Ifran. IFRAN.

6. HCEFLCD/DREFLCD-MA. (2007). Etude d'aménagement du BV de l'Oued Beht en amont du barrage El Kansera.

7. HCEFLCD. (1996a). P.N.A.B.V. Retrieved February 5, 2019, from http://www.eauxetforets.gov.ma/fr/text.aspx?id=1070\&uid=83

8. HCEFLCD. (1996b). Plan National d'Aménagement des Bassins Versants, Cadre stratégique. Maroc. Retrieved from http://www.eauxetforets.gov.ma/fr/text.aspx?id=1070\&uid=83

9. Jaouhari, M. (La vie éco/news. M. (2018, March 30). Envasement des 
$\begin{array}{llll}\text { barrages } & \text { au } & \text { Maroc. } & \text { Retrieved }\end{array}$ https://www.lavieeco.com/news/economie/envasement-des-barragesla-capacite-de-retenue-amputee-de-75-millions-de-m3-chaqueannee.html

10. Lakhili, F., Benabdelhadi, M., Chaouni, A., Bouderka, N., \& Lahrach, A. (2017). Cartographie de L'érosion qualitative des Sols du Bassin Versant De Beht ( Maroc ). American Journal of Innovative Research and Applied Sciences. ISSN 2429-5396 I, 174-185. Retrieved from www.american-jiras.com

11. Martin, J. (1982). Le Moyen Atlas Central. Etude géomorphologique. Revue de Géographie Alpine, 70(4), 444-444. Retrieved from: http://www.persee.fr/doc/rga_00351121_1982_num_70_4_2518_t1_0444_0000_1

12. Mesrar, H. (2016). Modélisation, quantification et définition des facteurs contrôlant le risque de l'érosion hydrique. Cas du bassin versant de l'oued Sahla, Rif central, Maroc. Université sidi Mohammed ben Abdellah,Faculté des Sciences, Dhar Mahraz, Fès. Maroc.

13. Michard, A. (1976). Eléments de Géologie Marocaine, 408.

14. Mimich, K., Essahlaoui, A., El Ouali, A., \& El Hmaidi, A. (2018). Using SWAT To Simulate A Moroccan Watershed, Including An Assessment Of The Most Sensitive Modelling Parameters With SUFI2, 50-63. https://doi.org/10.9790/1813-0710035063

15. Ouallali, A., Moukhchane, M., Aassoumi, H., \& Berrad, F. (2016). The Mapping of the Soils 'Degradation State by Adaptation the PAP / RAC Guidelines in the Watershed of Wadi Arbaa Ayacha ,. Journal of Geoscience and Environment Protection, 4(July), 77-88.

16. Ousmana, H., El Hmaidi, A., Essahlaoui, A., \& Bekri, H. (2017). Modélisation et cartographie du risque de l'érosion hydrique par l'application des SIG et des directives PAP / CAR . Cas du bassin versant de 1' Oued Zgane ( Moyen Atlas tabulaire, Maroc ). Bulletin de l'Institut Scientifique, Rabat, Section Sciences de La Terre, 39, 103-119.

17. PAP/CAR ( PAP/PAM/PNUE). (1998). PAP/CAR: Directives pour la cartographie et la mesure des processus d'érosion hydrique dans les zones côtières méditerranéennes. Split, Croatie.

18. Roose, E., Sabir, M., \& Laouina, A. (2010). Gestion durable de l'eau et des sols au Maroc: Valorisation des techniques traditionnelles méditerranéennes. Retrieved

from http://www.documentation.ird.fr/hor/fdi:010054911

19. SECE. Maroc. (2019a). Barrages en cours -département de l'Eau. Retrieved February

5 , 2019 , from 
http://81.192.10.228/patrimoine/barrages/barrages-en-cours/

20. SECE. Maroc. (2019b). Barrages existants - Département de l'Eau. Retrieved February 5, 2019, from http://81.192.10.228/patrimoine/barrages/barrages-existants/

21. Tribak, A., ELGarouani, A., \& Abahrour, M. (2012). L ' érosion hydrique dans les séries marneuses tertiaires du prérif oriental: agents , processus et évaluation quantitative. Revue Marocaine Des Sciences Agronomiques et Vétérinaires, 1(1), P 47-52. 